\title{
Regulation of inherently autoreactive VH4-34 B cells in the maintenance of human $B$ cell tolerance
}

\author{
Aimee E. Pugh-Bernard, ${ }^{1}$ Gregg J. Silverman, ${ }^{2}$ Amedeo J. Cappione, ${ }^{1}$ Michael E. Villano, ${ }^{3}$ \\ Daniel H. Ryan, ${ }^{4}$ Richard A. Insel, ${ }^{5}$ and Iñaki Sanz ${ }^{1}$
}

${ }^{1}$ Department of Medicine, University of Rochester Medical Center, Rochester, New York, USA

${ }^{2}$ Department of Medicine, University of California, San Diego, La Jolla, California, USA

${ }^{3}$ Department of Surgery,

${ }^{4}$ Department of Pathology, and

${ }^{5}$ Department of Pediatrics, University of Rochester Medical Center, Rochester, New York, USA

Address correspondence to: Iñaki Sanz, Department of Medicine, University of Rochester Medical Center, 601 Elmwood Avenue, Box 695, Rochester, New York 14642, USA.

Phone: (716) 275-2891; Fax: (716) 442-3214; E-mail: Ignacio_Sanz@URMC.rochester.edu.

Received for publication February 6, 2001, and accepted in revised form August 8, 2001.

\begin{abstract}
The study of human B cell tolerance has been hampered by difficulties in identifying a sizable population of autoreactive $B$ lymphocytes whose fate could be readily determined. Hypothesizing that $B$ cells expressing intrinsically autoreactive antibodies encoded by the VH4-34 heavy chain gene (VH434 cells) represent such a population, we tracked VH4-34 cells in healthy individuals. Here, we show that naive VH4-34 cells are positively selected and mostly restricted to the follicular mantle zone. Subsequently, these cells are largely excluded from the germinal centers and underrepresented in the memory compartment. In healthy donors but not in patients with systemic lupus erythematosus (SLE), these cells are prevented from differentiating into antibody-producing plasma cells. This blockade can be overcome ex vivo using cultures of naive and memory VH4-34 cells in the presence of CD70, IL-2, and IL-10. VH4-34 cells may therefore represent an experimentally useful surrogate for autoantibody transgenes and should prove valuable in studying human B cell tolerance in a physiological, polyclonal environment. Our initial results suggest that both positive and negative selection processes participate in the maintenance of tolerance in autoreactive human B cells at multiple checkpoints throughout B cell differentiation and that at least some censoring mechanisms are faulty in SLE.
\end{abstract}

J. Clin. Invest. 108:1061-1070 (2001). DOI:10.1172/JCI200112462.

\section{Introduction}

Understanding the mechanism(s) responsible for immunological tolerance in the B cell compartment is a fundamental question in immunology $(1,2)$. Transgenic models have been instrumental in understanding murine $\mathrm{B}$ cell tolerance (3-10) by providing a homogeneous population of transgenic $B$ cells of predetermined antigenic specificity, which enables investigators to ascertain the mechanisms of positive and negative selection that regulate autoreactive $B$ cells. In these models, tolerance can be mediated by mechanisms that operate at multiple checkpoints throughout B cell development including clonal anergy, clonal deletion, and receptor editing (11-13).

Yet, a great need for experimental approaches to the study of human B cell tolerance still exists. First, discrepancies between human and mouse B cell biology are well demonstrated by the different consequences that the loss of the Bruton's tyrosine kinase, intimately involved in B cell development and regulation, has in xid mice and XLA patients (14). Second, the physiological relevance of transgenic models suffers from the distortion of the $B$ cell repertoire and the inherent lack of competition with nonautoreactive B cells, which may be essential for some selection processes $(15,16)$.
The major stumbling block for the study of human B cell tolerance has been the identification of a sizable and homogeneous, autoantigen-specific B cell population whose fate and functional properties could be readily analyzed. We hypothesized that $\mathrm{B}$ cells expressing antibodies encoded by the $\mathrm{VH} 4-34$ heavy chain variable region gene (VH4-34 cells) could fulfill these requirements. Indeed, VH4-34-encoded antibodies (VH4-34 antibodies) are intrinsically autoreactive without requiring somatic mutation and independently of the associated light chains. The VH4-34 gene (formerly designated as VH4-21) (17) invariably conveys reactivity for conserved carbohydrate self-epitopes displayed at high density on red blood cells (RBCs) and other cell types. Virtually all VH4-34 IgM mAb's recognize the I/i RBC determinants that constitute the antigenic target of pathogenic autoantibodies in coldagglutinin (CA) disease (18-20). Strikingly, VH4-34 is a mandatory component of pathological anti-I/i cold agglutinins whether in idiopathic CA disease, lymphoproliferative disorders, or after infection with EpsteinBarr virus (EBV) or Mycoplasma pneumoniae (21-25).

These properties suggest that in order to prevent autoimmune disease, $\mathrm{VH} 4-34$ cells must be tightly regulated. Consistently, serum levels of VH4-34 antibodies 
account for only $0.5 \%$ of circulating Ig in normal donors but are elevated in patients with active systemic lupus erythematosus (SLE) (26). Moreover, in SLE serum VH434 antibodies correlate well with disease activity and visceral involvement and these antibodies can be found in kidney eluates (27-29). The inherent pathogenic potential of VH4-34 is further emphasized by the fact that, despite its disproportionate contribution to pathogenic autoantibodies, this variable region gene is not utilized in conventional protective antibodies (30-33).

In this study, we have tracked the expression of the VH4-34 gene segment throughout B cell differentiation. Our results demonstrate that VH4-34 cells are censored at multiple checkpoints during B cell development and are absent from the plasma cell (PC) compartment of healthy individuals but highly expressed in SLE plasma cells. Accordingly, we propose that inherently autoreactive VH4-34 cells can be viewed as a surrogate for autoantibody transgenes for the study of human B cell tolerance.

\section{Methods}

Antibodies and reagents. Antigen-presenting cell-conjugated (APC-conjugated) CD19 (SJ25C1), phycoerythrin-conjugated (PE-conjugated) CD27 (L128), streptavidin-PerCP (Becton-Dickinson Immunocytometry Systems, San Jose, California, USA); biotin-conjugated IgD (IA6-2), FITC-conjugated IgD (IA6-2), PE-conjugated CD38 (H1T2), FITC-conjugated CD20 (2H7), PE-conjugated CD23 (M-L233) (PharMingen, Los Angeles, California, USA); streptavidin-PE-Cy5 (DAKO Corp., Carpinteria, California, USA); PE-conjugated goat anti-rat IgM, PE-conjugated anti- $\kappa$ and anti- $\lambda$ $\mathrm{F}\left(\mathrm{ab}^{\prime}\right) 2$ (Southern Biotechnology Associates, Birmingham, Alabama, USA); goat anti-mouse IgG Alexa 488 (Molecular Probes Inc., Eugene, Oregon, USA). VH4-34 antibodies were detected with the rat monoclonal antiidiotypic antibody 9G4 (kindly provided by F.K. Stevenson, Tenovus Research Laboratories, Southampton, United Kingdom). The 9G4 antibody binds a crossreactive idiotype (CRI) that has been localized to the first framework region (FR1) of Ig heavy (H) chains encoded by the VH4-34 gene segment (34). Other VH4 antibodies were identified with mouse mAb LC1 that binds a CRI encoded by a subset of VH4 genes that does not include VH4-34 (35). Control VH3 antibodies were detected with avian monoclonal anti-idiotypic antibody LJ26, a recombinant single-chain Fv antibody specific for VH clan III products that recognizes the highly conserved framework regions FR1 and FR3 (36).

Human samples. Peripheral blood (PBL), bone marrow, and tonsil samples were obtained from healthy donors according to protocols approved by the University of Rochester Medical Center (URMC) Institutional Review Board. Only PBL was obtained from SLE patients. Patients were selected from the URMC Lupus Clinic if they had a clinical diagnosis of SLE and fulfilled four or more American College of Rheumatology criteria for the classification of SLE, had a SLEDAI index of 10 or more, and were treated with only antimalarials and/or low-dose prednisone ( $<10 \mathrm{mg} /$ day) at the time of venipuncture.

$B$ cell isolation. Heparinized PBL was collected from healthy donors and SLE patients. PBL B cells were obtained by magnetic positive selection using CD19 Microbeads (Miltenyi Biotec, Auburn, California, USA). Tonsils were obtained from 2- to 10-year-old patients after routine tonsillectomy. Tonsillar suspensions were subjected to one round of $\mathrm{T}$ cell depletion using 2-AET-SRBC (Colorado Serum Co., Denver, Colorado, USA). The resulting cells (> 98\% $\mathrm{CD} 19^{+}$) were used for phenotypic analysis by flow cytometry via a FACSCalibur or for sorting using a FACSVantage (both, Becton Dickinson Immunocytometry Systems, Mountain View, California, USA).

Plasma cell isolation. Bone marrow and tonsillar mononuclear cells isolated through a Ficoll-Paque gradient were labeled with CD138 (Syndecan-1) microbeads and positively selected on a MACS column (Miltenyi Biotec). The selected fractions were cytocentrifuged onto glass slides, dried and labeled for phenotypic analysis. PBL plasma cells from SLE patients were merely concentrated from the mononuclear cell layer by cytocentrifugation without previous positive selection.

Ex vivo culture of tonsil B cells and immunofluorescence detection of cytoplasmic ig's. Wells containing $1 \times 10^{6}$ sorted $\mathrm{IgD}^{+} / \mathrm{CD} 27^{-}$(naive) or $\mathrm{IgD}^{-} / \mathrm{CD}^{2} 7^{+}$(memory) tonsil B cells were cultured for 3 days with $10^{5}$ irradiated CD154transfected mouse fibroblasts and $10 \mathrm{U} / \mathrm{ml}$ of IL-2 (Peprotech Inc., Norwood, Massachusetts, USA) at $37^{\circ} \mathrm{C}$ with $5 \% \mathrm{CO}_{2}$. Viable cells were then cultured for 7 days with $10^{5}$ irradiated $\mathrm{CD} 70 / 300-19$ murine pre-B cells plus $10 \mathrm{U} / \mathrm{ml}$ of IL-2 and $100 \mathrm{ng} / \mathrm{ml}$ of IL-10 (Peprotech Inc.). The CD70/300-19 cell line was kindly provided by C. Morimoto (Dana Farber Cancer Institute, Boston, Massachusetts, USA). Subsequently, the cells were analyzed via FACSCalibur. Plasma cells were identified in preliminary experiments by accepted surface phenotypes including $\mathrm{CD} 38^{++} / \mathrm{CD} 20^{\mathrm{Lo}}$ and $\left(\mathrm{CD} 19^{+}, \mathrm{CD} 138^{+}, \mathrm{CD} 27^{++}\right)$, and their identity was confirmed by conventional morphological appearance under Giemsa staining (37-39). Given that virtually identical results were obtained with both surface phenotypes, only the CD38/CD20 scheme was systematically used for the experiments described here. Postculture plasma cells were sorted and analyzed for cytoplasmic antibodies. Briefly, $10^{5}$ cells were cytocentrifuged, fixed with $2 \%$ paraformaldehyde, and labeled with anti- $/ / \lambda F\left(a b^{\prime}\right) 2-P E$ and anti-CRI antibodies (9G4FITC or LJ26-biotin/streptavidin-FITC). The slides were analyzed by fluorescence microscopy (Olympus BX40, Olympus America Inc., Melville, New York, USA), and digitized images were stored on disk. Cells displaying intense PE cytoplasmic staining and typical morphology were counted as plasma cells. After counting a minimum of 500 plasma cells per culture, the frequency of VH4-34-expressing plasma cells was calculated as the percent of $\mathrm{PE}^{+}$plasma cells that also displayed intense 9G4-FITC staining. 
Immunocytochemistry studies. Tonsils were stained using a DAKO Corp. LSAB2 system according to the manufacturer's instructions. Briefly, $6-\mu \mathrm{m}$-thick acetone-fixed cryostat sections were incubated at room temperature for 10 minutes with primary antibodies (anti-CD20 antibody DAKO N1502, anti-CD3 antibody DAKO M0835, 9G4 or LC1). After rinsing with $1 \mathrm{X}$ PBS, sections were incubated for 10 minutes at room temperature with biotinylated anti-mouse or anti-rat Ig secondary antibodies, rinsed again, incubated with DAKO streptavidinperoxidase reagent for an additional 10 minutes, and developed using a diaminobenzidine chromogen solution for 10 minutes at room temperature. The slides were counterstained with hematoxylin for 2-5 minutes.

Flow cytometric analysis. Tonsil B cells were separated into three subsets: $\operatorname{sIgD}^{+} / \mathrm{CD} 38^{-}$cells (naive: $\mathrm{Bm} 1$ and $\mathrm{Bm} 2$ ), sIgD ${ }^{-} / \mathrm{CD}^{3} 8^{+}$cells (germinal center: $\mathrm{Bm} 3$ and $\mathrm{Bm} 4$ ), and sIgD ${ }^{-} / \mathrm{CD}^{-} 8^{-}$cells (memory: Bm5) (40). To that end, the cells were labeled with CD38-PE/IgDbiotin followed by streptavidin-PE-Cy5 and 9G4-FITC (for VH4-34 antibodies) or CD38-PE/IgD-FITC and LJ26-biotin followed by streptavidin-PE-Cy5 (for VH3 antibodies) and analyzed with a FACSCalibur as described elsewhere (41). Naive cells were further fractionated into $\mathrm{Bm} 1$ and $\mathrm{Bm} 2$ by expression of $\mathrm{CD} 23$, and germinal center cells were classified as $\mathrm{Bm} 3$ or $\mathrm{Bm} 4$ based on the expression of CD77. Briefly, cells were stained with CD38-cychrome and IgD-biotin/streptavidin-APC followed by CD23-PE or CD77-PE, and 9G4FITC or CD38-cychrome and IgD-FITC followed by CD23-PE or CD77-PE and LJ26-biotin/streptavidinAPC and analyzed via FACSCalibur.

To detect potential differences in the global level of surface immunoglobulin (sIg) on each specific subset, cells were labeled with CD38-PE and IgD-biotin/streptavidin-PE-Cy 5 followed by anti- $\kappa / \lambda \mathrm{F}\left(\mathrm{ab}^{\prime}\right)_{2}$-PE antibodies. To determine the relative intensity of sIg on VH4-34 and VH3 populations, the cells were stained with CD19-PE and either 9G4-FITC or LJ26biotin/streptavidin-FITC. Moreover, histogram analysis of the frequency of VH4-34 cells in each B cell subset was performed in parallel with appropriate isotype control antibodies. In all cases, the gates were set so that $0.5-1.0 \%$ of cells stained with isotype control fell to the right of the cursor. This maneuver ensures that any cells staining weakly with 9G4 were considered as positive in the final analysis. Given the large numbers of VH3 cells present in all compartments, gates for the LJ26 histograms were set by visual inspection.

PBL B cells were fractionated into naive cells $\left(\mathrm{CD} 27^{-} / \mathrm{IgD}^{+}\right)$and memory cells $\left(\mathrm{CD}^{2} 7^{+} / \mathrm{IgD}^{-}\right.$or $\left.\mathrm{CD}^{2} 7^{+} / \mathrm{IgD}^{+}\right)(42)$. Briefly, purified $\mathrm{B}$ cells were labeled with CD19-APC, CD27-PE, and either IgD-biotin/streptavidin-PerCP/9G4-FITC or IgD-FITC/LJ26-biotin/streptavidin-PerCP and analyzed on a FACSCalibur.

To detect intracellular expression of antibody, cells were fixed with $2 \%$ paraformaldehyde, permeabilized with buffer containing $0.1 \%$ saponin, and labeled with 9G4-FITC or LJ26-biotin/streptavidin-PE-Cy5.
Statistical significance was assessed using nonparametric Mann-Whitney $U$ tests with the GraphPad Prism software (GraphPad, San Diego, California, USA). The CellQuest software (Becton Dickinson Immunocytometry Systems) was used to calculate the frequencies of $B$ cell subsets.

$P C R$. Genomic DNA was isolated using the EasyDNAKit (Invitrogen Corp., Carlsbad, California, USA) from tonsil B cells, the Ramos B cell lymphoma (which expresses a rearranged $\mathrm{VH} 4-34 \operatorname{IgM}$ ) and the Jurkat acute $T$ cell leukemia. Genomic DNA served as template for the amplification of $\mathrm{VH} 4-34-\mathrm{D}-\mathrm{J}_{\mathrm{H}}$-specific gene rearrangements. For each sample, 33, 100, and $300 \mathrm{ng}$ of genomic DNA were amplified using a primer specific for the leader intron of VH4-34 (5'-CCAGACGTGAAGATATGGGA-3') and two $\mathrm{J}_{\mathrm{H}}$ primers that anneal with more than $98 \%$ identity to all 12 allelic forms of the six human $\mathrm{J}_{\mathrm{H}}$ genes (5'-TGAGGAGACRGTGACCAGGGT-3' and $5^{\prime}$-TGAaGAGACGGTCATTGT-3'). Amplification consisted of an initial denaturation step of 2 minutes at $94^{\circ} \mathrm{C}$ followed by $20-35$ cycles of 1 minute at $94^{\circ} \mathrm{C}$; 1.5 minutes at $60^{\circ} \mathrm{C}$; and 1.5 minutes at $72^{\circ} \mathrm{C}$; with a final extension step of 4 minutes at $72^{\circ} \mathrm{C}$. Amplified products were visualized by electrophoresis through $2 \%$ Nusieve agarose containing $1 \mu \mathrm{g} / \mathrm{ml}$ ethidium bromide. The PCR product signal was quantified by densitometry using Eagle Eye II/EagleSight software (Stratagene, La Jolla, California, USA).

\section{Results}

In bealthy individuals, VH4-34 cells are predominantly expressed in the naive repertoire and are underrepresented in the germinal center and memory compartments. Based on our working hypothesis, intrinsically autoreactive VH434 cells should be censored during B cell development. Previous studies estimated that $2-10 \%$ of total B cells express VH4-34. However, these studies were at best semiquantitative PCR assessments of the frequency of VH4-34 in $\mathrm{C} \mu$ and $\mathrm{C} \gamma$ transcripts and did not differentiate among discrete $B$ cell subsets $(26,43,44)$. To elucidate this issue we examined the distribution of VH434 cells within purified tonsillar B cell subsets and determined that, on average, $80-90 \%$ of VH4-34 cells had a naive $(\mathrm{N})$ phenotype, compared with $5 \%$ and $7 \%$ with a germinal center (GC) and memory (M) phenotypes, respectively (Figure 1 and Table 1). VH4-34 cells made up to $10 \%$ of tonsil naive B cells, but only $0.5-2.0 \%$ of germinal center and memory B cells. Similar distributions were observed in the PBL naive and memory compartments. As opposed to VH4-34 cells, the relative frequency of $\mathrm{B}$ cells expressing $\mathrm{VH} 3$ genes was conserved across the different developmental compartments (Figure 1, a and b). Thus, $\mathrm{LJ}^{2} \mathrm{6}^{+}$cells represented approximately one third of total tonsil $\mathrm{N}$ cells, one fourth of GC cells, and up to one half of M cells.

Tonsil naive $B$ cells include a virgin subset $(\mathrm{Bm} 1)$ and a ligand selected subset $(\mathrm{Bm} 2)$, the latter population being characterized by the acquisition of surface $\mathrm{CD} 23$ (40). Analysis of these subsets revealed an statistically 

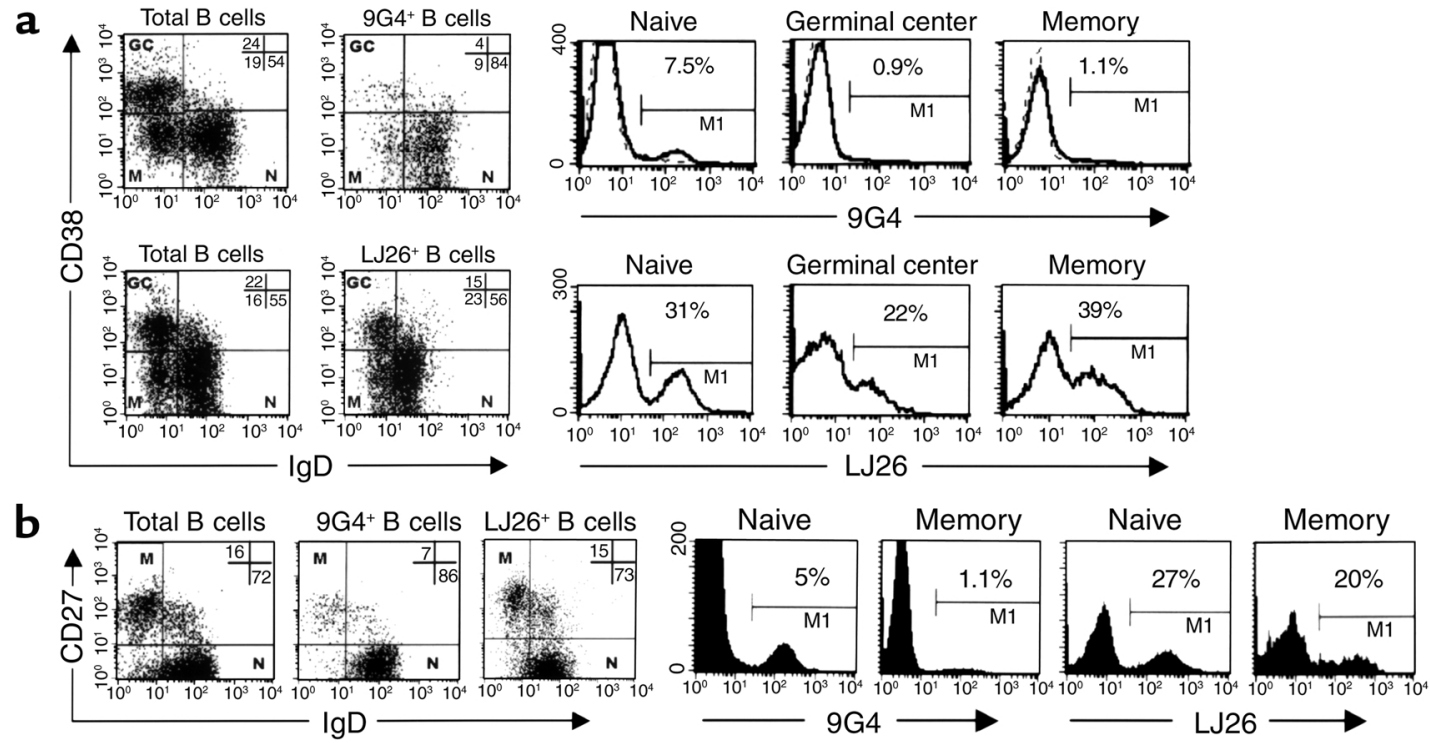

C
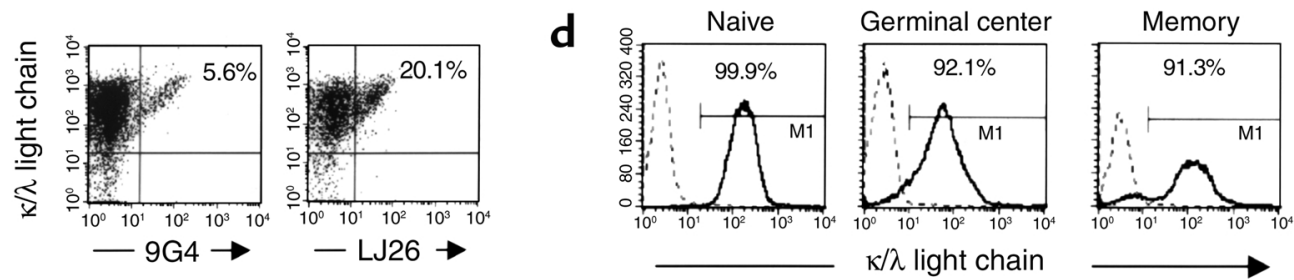

$\mathbf{e}$
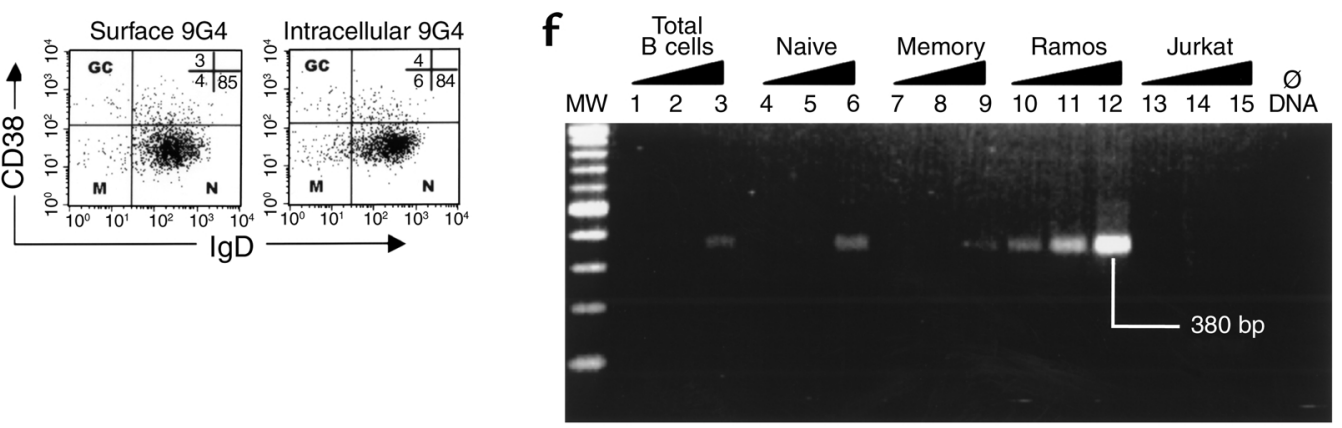

\section{Figure 1}

Representation of VH4-34 cells in human B cell subsets. (a) Distribution of total, VH4-34 (9G4) and VH3 (LJ26) B cells into naive (N), germinal center (GC), and memory (M) subsets in a representative tonsil. Histograms demonstrate the frequency of $9 G 4$ and LJ26 cells in each subset. Bold lines represent VH4-34 and VH3 cells. Isotype antibody controls for $9 \mathrm{G} 4$ are shown as superimposed dashed lines. (b) Similar

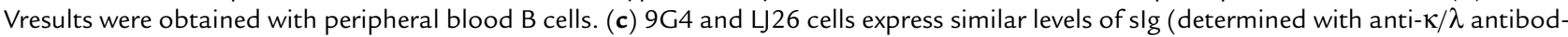
ies). (d) Analysis of the slg expression by tonsil B cells. At least $90 \%$ of cells in each subset express detectable levels of slg. (e) Tonsil B cells were permeabilized and analyzed for either surface or cytoplasmic expression of VH4-34. (f) PCR analysis of VH4-34 rearrangements. Samples are as follows: MW, 100 bp ladder; $\varnothing$ DNA, negative control without input DNA; 1-3, total tonsil B cells; 4-6, naive B cells; 7-9, memory B cells; 10-12, VH4-34-expressing Ramos B cell lymphoma; 13-15, Jurkat T cells. The following amounts of DNA template were used: lanes 1, 4, 7, 10, and 13 (33 ng); lanes 2, 5, 8, 11, and 14 (100 ng); lanes 3, 6, 9, 12, and 15 (300 ng). Noticeable amplification of rearranged VH4-34 was detected in memory cells only after 35 PCR cycles with the highest amount of template DNA (300 ng). The amount of product obtained with memory cells was four to ten times lower than that obtained with naive B cells (average of four experiments). Calculations based on equivalent amounts of Ramos cells or Ramos DNA indicate that less than 2\% of memory cells express VH4-34.

significant expansion (up to fourfold) of VH4-34 cells from the $\mathrm{Bm} 1$ to $\mathrm{Bm} 2$ stage (Figure $2 \mathrm{a}$ and Table 1 ). It thus appears that VH4-34 cells are preferentially found and even positively expanded within the naive compartment. These findings were corroborated by immunocytochemical staining of tonsils that showed $9 \mathrm{G} 4^{+}$cells to be concentrated in the follicular mantle and largely excluded from the germinal center struc- tures (Figure $2 \mathrm{~b}$ ). This anatomical restriction was conserved in 50 individual germinal centers obtained from two independent tonsils. In contrast, B cells expressing surface $\mathrm{VH} 4$-encoded antibodies recognized by the anti-idiotype LC 1 were found in $40 \%$ of all germinal centers studied.

The first caveat is that the GC subset contains both sIg centroblasts (Bm3) and $\operatorname{sIg}^{+}$centrocytes $(\mathrm{Bm} 4)$, and, 
therefore, surface staining would not detect the sIgfraction of this population (40). To address this problem we analyzed four independent samples of GC cells for their expression of sIg using anti-light chain antibodies and found that only $10-16 \%$ of this population was negative for $\operatorname{sig}$ (data not shown). This observation would be consistent with a relative scarcity of ongoing GC reactions in donors without acute tonsillitis and suggests that sIg- GC cells did not significantly impact our results. Furthermore, intracellular staining of total B cells with 9G4 yielded similar results to those obtained by surface staining (Figure 1e). Finally, when GC cells were subdivided into the $\mathrm{Bm} 3$ and $\mathrm{Bm} 4$ subsets, intracellular staining demonstrated that VH4-34 cells represent less than $1 \%$ of the $\mathrm{sIg}^{-} \mathrm{Bm} 3$ subset (Table 1 ). The second caveat is that the GC and M subsets could contain VH4-34 cells that no longer express the 9G4 idiotype as a result of somatic hypermutation. Indeed, somatic mutation does occur outside the conventional antigen-binding site and can also affect the framework regions. Yet several lines of evidence strongly argue against this possibility. (a) It has been previously reported that the 9G4 idiotype is highly tolerant to somatic point mutations $(31,45)$. (b) Similar reductions to the ones observed for $9 \mathrm{G} 4$ were not detected in our experiments for two other idiotypes that should have been equally affected by somatic hypermutation (LJ26 and LC1). Indeed, as discussed above, we frequently found $\mathrm{LC}^{+}$germinal centers in the absence of $9 \mathrm{G} 4^{+}$cells. (c) Given that $9 \mathrm{G} 4^{+}$plasma cells were readily detected in patients with SLE but absent in normals (see below), it is highly unlikely that their absence in healthy subjects would result from failure to detect a mutated idiotype. Finally, we determined by PCR the relative expression of rearranged $\mathrm{VH} 4-34$ in genomic DNA extracted from the different $B$ cell subsets (Figure 1f). The PCR results parallel the flow cytometry data and are consistent with a significantly reduced frequency of VH4-34 cells in GC and M subsets. As an additional control, we also tried to amplify V(D)J rearrangements containing VH4-34 from sorted 9G4- M cells and obtained a very faint band only after 45 cycles of amplification, a result consistent with either a very low frequency of $9 \mathrm{G} 4^{-} / \mathrm{VH} 4-34^{+} \mathrm{M}$ cells or with a low level of contamination with $9 \mathrm{G}^{+}$cells (data not shown).

VH4-34 is absent from bone marrow and tonsil plasma cells of healthy individuals. Despite the substantial representation of VH4-34 cells in the peripheral B cell compartment, VH4-34 antibodies contribute on average less than $0.5 \%$ of serum IgM and $\operatorname{IgG}(31,43)$. This discrepancy could be explained by postulating that VH4-34 cells are prevented from differentiating into antibodysecreting plasma cells (PCs). Indeed,

Table 1 this hypothesis would be consistent with the observation that VH4-34 has never been found expressed in multiple myelomas (46). However, the expression of VH4-34 in normal PCs has never been formally tested. Therefore, we analyzed approximately 5,000 bone marrow PCs from 12 healthy donors (an average of 400 PCs per donor) without finding a single instance of cytoplasmic expression of VH4-34 antibodies (Figure 3). In contrast, control antibodies encoded by other VH4 genes or by $\mathrm{VH} 3$ genes were expressed by $17-23 \%$ and $25-33 \%$, respectively, of all PCs analyzed. Similar results were obtained from 1,200 tonsillar PCs from three donors (an average of 400 PCs per donor) selected on the basis of their surface expression of CD138. Despite the complete absence of expression of $\mathrm{VH} 4-34$, control $\mathrm{VH} 4$ and $\mathrm{VH} 3$ antibodies were expressed in $4-7 \%$ and in $15-22 \%$ respectively of tonsillar PC. Our results suggest the presence within a healthy immune environment of a final checkpoint that prevents terminal differentiation of residual autoreactive VH4-34 B cells. Presumably, bone marrow samples contain a higher frequency of late, long-lived plasma cells, whereas tonsils would have mostly early, short-lived plasma cells (47). Therefore, our results would be consistent with an early block in PC differentiation. The mechanism(s) responsible for this block is still unknown, both in humans and mice, but it might include autoantigen-mediated effects and/or T cell-dependent mechanisms (11).

$V H 4-34$ is present in plasma cells of patients with SLE. It has been shown that elevated serum levels of VH4-34 antibodies are found in $70 \%$ of lupus patients and correlate with disease activity $(27,29)$. Therefore, we studied SLE patients to determine whether in an autoimmune environment VH4-34 cells are allowed to differentiate into plasma cells. Early plasma cells are readily found in the PBL of active SLE patients and most likely represent the initial progeny of activated B cells mobilized from secondary lymphoid organs on their way to the bone marrow $(37,39)$. Of approximately 1,600 PCs analyzed from eight donors (an average of 200 per donor), $5-20 \%$ were shown to contain VH4-34 antibodies, which were in vir-

Relative distribution of VH4-34 cells in discrete human B cell subsets

\begin{tabular}{llccc}
\hline Samples & Subsets & $\begin{array}{c}\text { Frequency of } \\
\text { each subset }^{\mathrm{A}}\end{array}$ & $\begin{array}{c}\text { Frequency of } \\
\text { VH4-34 (9G4) in } \\
\text { each subset }^{\mathrm{B}, \mathrm{C}}\end{array}$ & $\begin{array}{r}\text { Frequency of } \\
\text { VH3 (L)26) in } \\
\text { each subset }^{\mathrm{B}, \mathrm{C}}\end{array}$ \\
Tonsil & Naive $(\mathrm{Bm} 1+\mathrm{Bm} 2)$ & $53 \pm 11$ & $7.3 \pm 3.1$ & $33 \pm 2.2$ \\
$n=15$ & GC (Bm3 $+\mathrm{Bm} 4)$ & $17 \pm 9$ & $1.2 \pm 0.5^{\mathrm{B}}$ & $24 \pm 6.7$ \\
& Memory $(\mathrm{Bm5})$ & $21 \pm 9$ & $1.6 \pm 0.6^{\mathrm{B}}$ & $43.3 \pm 6.9$ \\
Tonsil & Naive (Bm1) & $10 \pm 5$ & $4.1 \pm 2.4$ & $31.4 \pm 4.5$ \\
$n=8$ & Naive (Bm2) & $31 \pm 10$ & $7.0 \pm 2.6^{\mathrm{C}}$ & $33.8 \pm 4.1$ \\
& Centroblasts (Bm3) & $14 \pm 7$ & $0.7 \pm 0.4^{\mathrm{B}}$ & $21.9 \pm 2.0$ \\
& Centrocytes $(\mathrm{Bm} 4)$ & $25 \pm 12$ & $1.8 \pm 0.9^{\mathrm{B}}$ & $17.1 \pm 2.5$ \\
$\mathrm{PBL}$ & Naive & $68 \pm 8$ & $5.1 \pm 2.2$ & $29.0 \pm 5.3$ \\
$n=7$ & Memory & $29 \pm 7$ & $1.3 \pm 0.3^{\mathrm{B}}$ & $24.0 \pm 4.1$
\end{tabular}

${ }^{A}$ All values are expressed as mean percentage \pm SD. ${ }^{B} P<0.05$ when germinal center and memory subsets are compared with naive cells under the same column (9G4 or LJ26). ${ }^{C} P<0.05$ for Bm1-to-Bm2 or $\mathrm{Bm} 3$-to-Bm4 comparisons under the same column (9G4 or LJ26). Tonsil B cell subsets: Bm1 (IgD+ CD38- CD23-), Bm2 (IgD ${ }^{+}$CD38- CD23+ $), B m 3\left(\operatorname{lgD}^{-} \mathrm{CD} 38^{+} \mathrm{CD}^{-} 7^{+} \mathrm{slg}^{-}\right), \mathrm{Bm} 4\left(\operatorname{lgD}^{-} \mathrm{CD} 38^{+} \mathrm{CD} 77^{-}\right.$ $\left.\mathrm{slg}^{+}\right), \mathrm{Bm} 5\left(\operatorname{lgD} \mathrm{D}^{-} \mathrm{CD} 38^{-}\right)$. PBL subsets: naive $\left(\lg \mathrm{D}^{+} \mathrm{CD} 27^{-}\right)$, memory $\left(\operatorname{lgD} \mathrm{D}^{-} \mathrm{CD} 27^{+}\right.$plus $\left.\lg \mathrm{D}^{+} \mathrm{CD} 27^{+}\right)$. 

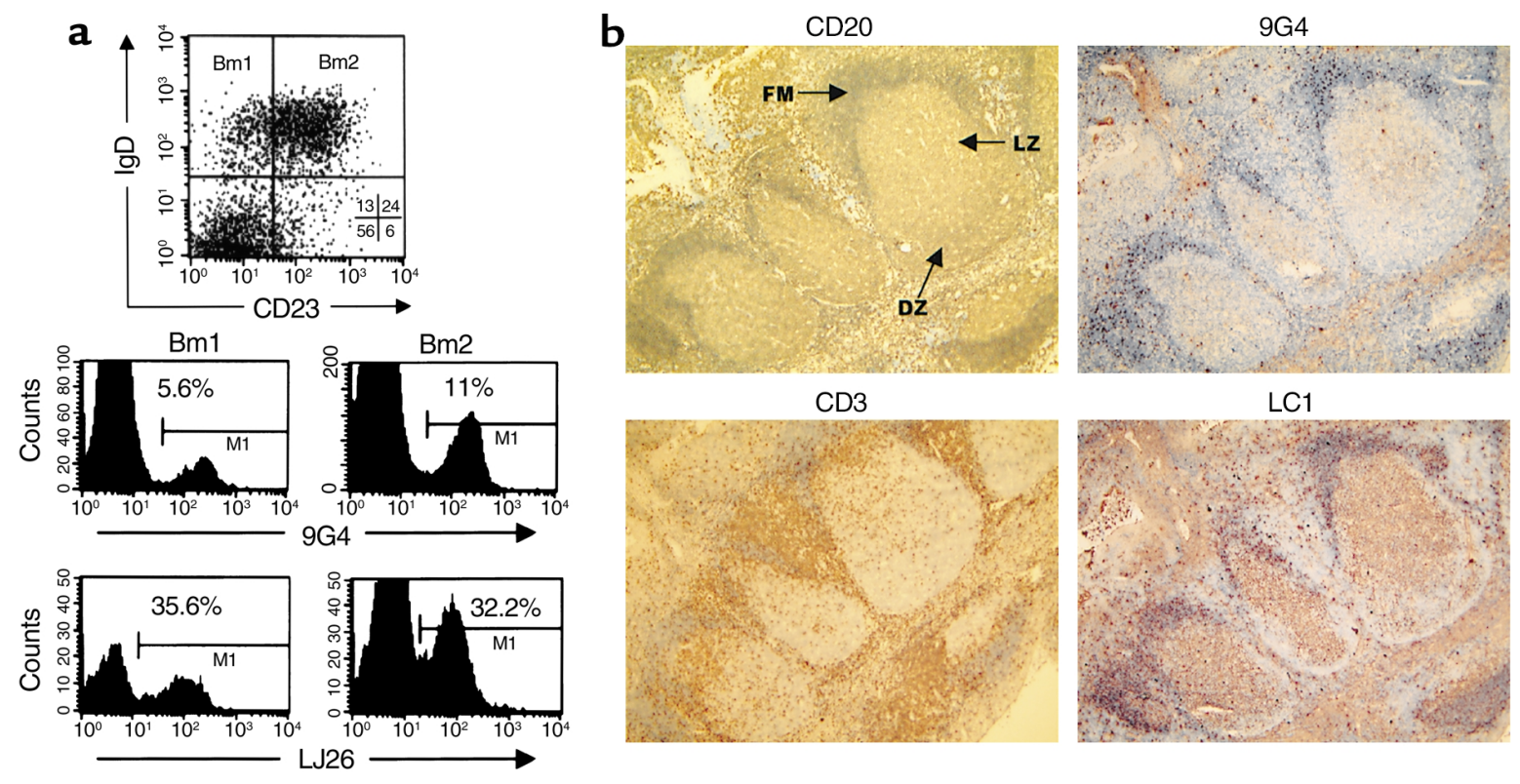

Figure 2

$\mathrm{VH} 4-34$ cells primarily reside within the follicular mantle zone. (a) Purified tonsil naive B cells were fractionated into Bm1 and Bm2 subsets based on their expression of surface IgD and CD23 (dot plot on the left) and stained with 9G4 or LJ26 (histograms on the right). A two- to fourfold expansion of $\mathrm{VH} 4-34$ cells from the $\mathrm{Bm} 1$ (virgin naive) to the $\mathrm{Bm} 2$ (ligand-selected naive) compartment was consistently demonstrated in all samples analyzed. (b) Immunohistochemical staining of adjacent tonsil sections was performed with antibodies against $B$ cells (anti-CD20), VH4-34 cells (9G4), VH4 cells (LC1), or T-cells (anti-CD3). A conventional distribution of B cells and T cells was observed within the follicular mantle (FM), dark zone (DZ), and light zones (LZ) of typical germinal center structures. VH4-34 cells were concentrated in the FM and were virtually absent from the germinal center proper. In contrast, B cells expressing VH4 antibodies distinct from $\mathrm{VH} 4-34$ were readily visualized inside the germinal centers (LC1 staining).

tually all instances of the IgG isotype (Figure 3). In contrast, in keeping with previous studies showing that increased serum 9G4 levels are 95\% specific for SLE and are absent from other autoimmune diseases including Sjögren syndrome $(27,29)$, PBL PCs from four rheumatoid arthritis patients were also devoid of VH4-34 antibodies (data not shown). These findings are consistent with the notion that the autoimmune process underlying SLE results in a disruption of the censoring mechanisms that normally prevent autoreactive $B$ cells, in this case represented by VH4-34 cells, from differentiating into plasma cells that autonomously secrete large amounts of autoantibodies. Furthermore, the preferential production of IgG antibodies by VH4-34 lupus plasma cells would explain the fact that cold agglutinininduced hemolytic anemia is rare in SLE (48).

Normal VH4-34 cells can differentiate into plasma cells ex vivo. The intrinsic differentiation potential of VH4-34 cells was tested ex vivo under culture conditions that have been shown to induce plasma cell development $(38,49)$. First, tonsil B cells were sorted into either $\mathrm{N}$ or $\mathrm{M} \mathrm{B}$ cells and cultured for 2-3 days with CD154-transfected murine fibroblasts in the presence of IL-2. This step induces the activation of naive cells, which can result in a slight induction of surface CD27. Subsequently, CD154-expressing adherent cells were eliminated, and the cultures were continued for 5-7 days with $\mathrm{CD} 70$-expressing murine pre-B cells in the presence of IL-2 and IL-10. The CD70-CD27 interaction plays an important role in B cell activation and has been shown to increase the percentage of cells with a plasma cell phenotype (38).

Our results (Figure 3 ) reveal that B cells cultured under these conditions do indeed generate cells with the morphologic appearance and the phenotypic profile of plasma cells (CD38 $\left.{ }^{\text {hi }} / \mathrm{CD} 20^{\text {lo }}\right)(37)$. Based on five independent tonsils, postculture frequencies were significantly higher than baseline values, thus indicating successful de novo generation of plasma cells from both naive and memory cell cultures. As expected, significantly higher numbers of plasma cells were obtained from memory cells than from naive cells (mean \% \pm SD: $33.16 \pm 3.12$ and $6.0 \pm 1.78$, respectively; $P<0.001)$. The percent of plasma cells obtained with naive cell cultures was also significantly higher than the preculture values $(2.08 \pm 0.49 ; P<0.01)$. Consistently with the relative abundance of VH4-34 cells in the naive and memory subsets, a higher frequency of plasma cells expressed cytoplasmic VH4-34 antibodies in naive cultures than in memory cultures $(15 \pm 2.0$ and $5.2 \pm 1.3$, respectively; $P=0.01$ ).

\section{Discussion}

Our results support the concept that in a healthy immune system the majority of VH4-34 cells are concentrated in the $\mathrm{N}$ repertoire, are scarcely represented in the GC and $\mathrm{M}$ cell compartments, and are excluded from the PC compartment. This skewed distribution was not 
a

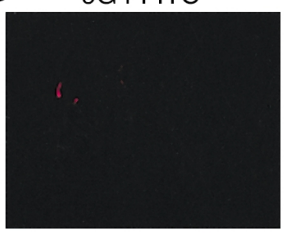

b

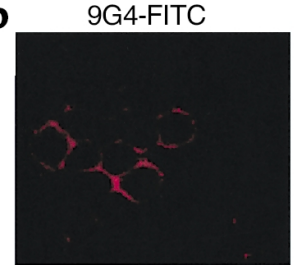

C

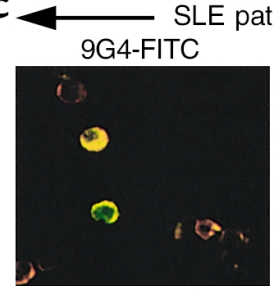

Anti- $/ \lambda-$ RPE

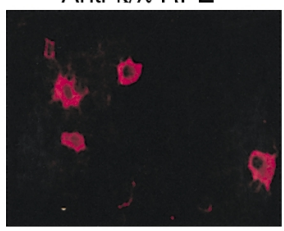

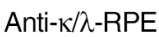

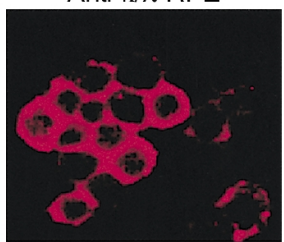

MV

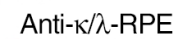

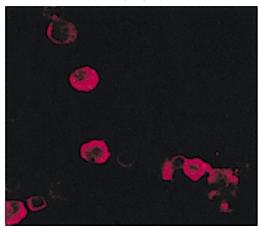

LJ26-FITC

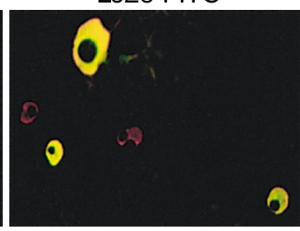

LJ26-FITC
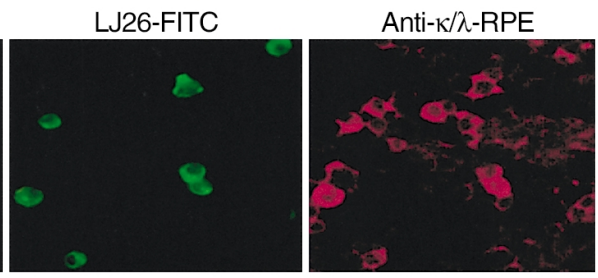

SLE patient JS

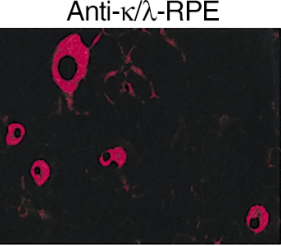

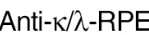

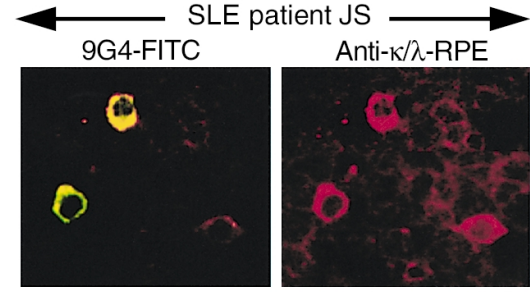

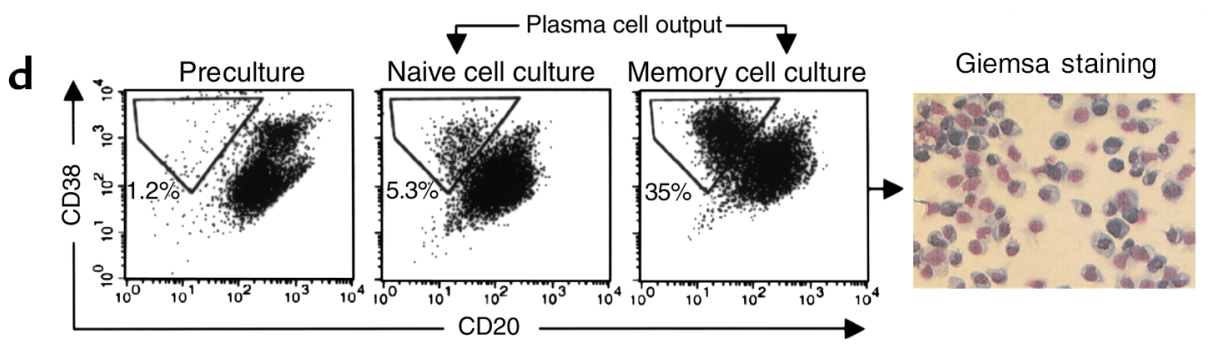

e

Tonsil naive B cells

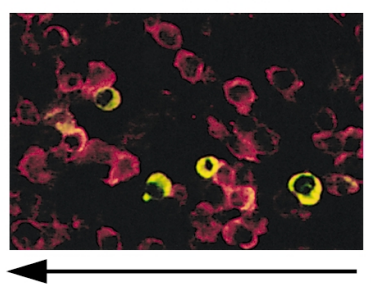

9G4-FITC

\section{Figure 3}

Expression of VH4-34 antibodies in plasma cells from healthy donors and SLE patients. (a) Bone marrow plasma cells from healthy donors were enriched using a MACS CD138 column, and the cytoplasmic expression of VH4-34 or VH3 antibodies was detected with anti-light chain and VH-specific anti-Id antibodies 9G4, LJ26, or LC1 (data not shown). In contrast to VH3 and other VH4 antibodies, expression of VH4-34 was absent from normal plasma cells. (b) Identical results were obtained with tonsillar cells. (c) Plasma cells from the peripheral blood of active SLE patients express $\mathrm{VH} 4-34$ antibodies with significant frequency (two representative examples are shown). Further analysis revealed that more than $95 \%$ of VH4-34 antibodies were of the IgG isotype (data not shown). (d) Differentiation of VH4-34 B cells into plasma cells in vitro. Naive and memory tonsil B cells were sorted and independently cultured in the presence of CD70, IL-2, and IL-10 as described. The frequency of plasma cells was determined by flow cytometry before fractionation (left panel) and after culture (middle panels), and morphological confirmation was obtained by Giemsa staining (right panel). Significant numbers of plasma cells with a CD $38^{\mathrm{Hi}} / \mathrm{CD} 20^{\mathrm{Lo}}$ phenotype were obtained with naive and memory cell cultures. (e) Plasma cells obtained in culture were analyzed for intracellular expression of VH4-34 antibodies using FITC-conjugated $9 \mathrm{G} 4$ and anti-K/ $\lambda$-RPE. VH4-34+ plasma cells were detected at a frequency consistent with the relative abundance of VH4-34 cells in the initial samples and therefore were significantly higher in the naive cell cultures than in the memory cell cultures.

observed in control VH3 B cells nor in cells expressing other VH4 antibodies. We believe that this difference reflects the unique properties of the VH4-34 gene segment. Indeed, VH4-34 encodes pathogenic autoantibodies in the germline configuration without the contribution of somatic mutation and to a large extent independently of somatically generated third hypervari- able regions (HCDR3) and of associated light chains. These properties would endow early VH4-34 cells with a rather homogeneous phenotype rendering them susceptible to selection through interaction with ubiquitous self-antigens such as the $\mathrm{I} / \mathrm{i}$ epitopes abundantly expressed in RBCs as well as in lymphocytes and other tissues (50-53). This notion would be consistent with 
the observation that in healthy individuals, VH4-34 antibodies are not selected during the $\mathrm{T}$ ell dependent, antigen-driven maturation of protective immune responses (30-33). In contrast, as a whole, the human antibody repertoire is characterized by the repeated utilization of a handful of "conventional" VH genes that are utilized both by protective and autoimmune responses alike, with the ultimate antigenic reactivity being determined by the presence of specific HCDR3 regions, antigen-driven somatic mutations, and associated light chains (30, $32,54)$. As a consequence, autoreactive B cell clones expressing most $\mathrm{VH}$ genes should represent a minor fraction of the global antibody repertoire encoded by those genes; therefore their elimination would not result in significant quantitative changes.

The identification of a regulated human B lymphocyte subset with intrinsically autoreactive properties raises a number of interesting questions and establishes an experimental system that enables investigators to study the mechanisms of B cell tolerance in a physiological, polyclonal environment. While it might have been expected that a gene with great pathogenic potential should have been deleted over evolutionary time, VH4-34 is not only universally present in the human germline without any polymorphism, but it is also highly expressed in the preimmune B cell repertoire (17). Thus, we found that the expression of VH4-34 (one of 50 functional genes in the $\mathrm{VH}$ locus) in the $\mathrm{N}$ is higher than it would expected in a stochastic model based on genomic complexity. Our observation of a significant expansion of $\mathrm{N} \mathrm{VH4-34} \mathrm{cells} \mathrm{in} \mathrm{the} \mathrm{Bm2} \mathrm{sub-}$ set (which contains activated, ligand-selected cells) strongly suggests that positive selection could account for such overrepresentation. Positive selection of VH4-34 cells would require an explanation regarding the mechanisms and purpose of expanding a potentially pathogenic population and would also demand the existence of concurrent safety mechanisms to prevent the development of overt autoimmunity.

Even in transgenic mice, the process of positive selection remains to be conclusively defined. However, several studies strongly suggest a role for positive selection in the B cell compartment (55-60). More conclusively perhaps is the recent demonstration that anti-Thy-1 antibodies are positively selected in Thy- 1 transgenic mice (61). These models postulate that strong signaling triggered through engagement of the $\mathrm{B}$ cell receptor complex by high-density multivalent antigens may induce differentiation into the B1 lineage $(60,62)$. In the mouse, it has been proposed that antigen-induced differentiation into long-lived peritoneal B1 cells and expression of CD5 may help maintain tolerance in autoreactive $B$ cells (63).

Interestingly, most of the aforementioned examples of positive selection involve self-reactive $\mathrm{B} 1$ cells and natural autoantibodies that recognize carbohydrate determinants and play an important role in natural immunity. Of note, VH4-34 antibodies appear to share many such features, including their preference in germline configuration for carbohydrates expressed at high density, and their preferential expression as IgM antibodies in either EBV and Mycoplasma pneumoniae infections, in idiopathic CA disease, and in lymphoid malignancies. It is therefore tempting to speculate that the underrepresentation of VH4-34 cells in germinal centers and germinal center-derived B cells and plasma cells could be the result of their positive selection into a separate anatomic and functional compartment, perhaps a human "B1-like" compartment. Such interpretation is consistent with the observation that VH4-34 antibodies are frequently made by human cord blood $\mathrm{CD}^{+} \mathrm{B}$ cells but are absent from CD5- cells (64). Yet, this dichotomy is not preserved in the adult and singlecell PCR analysis of adult IgM B cells has demonstrated similar expression of $\mathrm{VH} 4-34$ in $\mathrm{CD}^{+}$and $\mathrm{CD}^{-}$ cells (65). CD5, however, may not be a good marker of $\mathrm{B} 1$ cells in many species, and human $\mathrm{CD}^{+} \mathrm{B}$ cells differ from their murine counterpart in several aspects including the presence of somatic mutations and the expression of junctional diversity $(66,67)$. Elucidation of this issue therefore will require a clearer working definition of human B1 cells.

Alternatively, VH4-34 cells could be positively selected by either microbial or self-antigens into the spleen marginal zone (MZ), as it has been demonstrated in VH81X transgenic mice (57). Similar to our results with 9G4, transgenic VH81X B cells are mostly (albeit not completely) absent from the germinal centers, and the idiotype preferentially expressed by MZ cells (VH81X/Vк1C) cannot be detected in serum or in mature plasma cells.

In the current study, we found that VH4-34 cells accumulate as $\mathrm{Bm} 2$ naive cells in the mantle zone of tonsillar secondary follicles. To the best of our knowledge, the differentiation potential of human $\mathrm{Bm} 2$ cells remains to be formally tested, but, presumably, they become part of the recirculating follicular cell pool from which $\mathrm{MZ}$ $B$ cells are recruited (68). Indeed, the known biologic properties of MZ B cells would make them prime candidates to account for the fact that up to $86 \%$ of patients infected with Mycoplasma pneumoniae experience a fast production of IgM anti-I cold agglutinins lasting less than 4 weeks (69-71). Along with our culture experiments, these observations strongly suggest that VH4-34 cells persist at a substantial level in the functional repertoire of healthy individuals and that under appropriate stimulation they are capable of generating vigorous but self-limited plasma cell responses. Experiments are currently under way in our laboratory to determine the distribution of VH4-34 cells in the different compartments of the spleen.

We propose a beneficial role for VH4-34 antibodies in the normal homeostasis of the immune system. Although the ultimate reason for its positive selection is still unknown, important clues are available. Thus, while virtually all VH4-34 mAb's recognize RBC carbohydrate antigens, many such antibodies cross-react with other nonprotein antigens such as bacterial LPS, DNA, and tumor gangliosides (72-74). In our working 
model, one could envision surface VH4-34 antibodies as a type of innate receptor that could recognize repetitive patterns present in self- and microbial antigens $(75,76)$. Under conditions that provide strong stimulation these cells could differentiate into MZ cells and play a protective role either by clearing self-antigens, by swiftly reacting with bacterial antigens or by maintaining peripheral $\mathrm{T}$ cell tolerance through the presentation of self-antigens. The required stimulation could be provided by several factors including the nature of the antigen and the engagement of appropriate coreceptors and signaling pathways (77). The highest expression of the B cell-specific Toll-like receptor RP105, which is required for responsiveness to LPS via TLR4, is found in humans in the mantle zones where VH4-34 cells are concentrated, suggesting that innate receptors may play a role in selecting these cells (78). On the other hand, these cells would have to walk a tight rope to avoid being recruited into the $\mathrm{T}$ cell-dependent $\mathrm{B} 2$ memory repertoire with the potential to produce autoimmune disease $(76,79,80)$. Indeed, it could be envisioned that intrinsic $B$ cell abnormalities might push these cells through an undesired and detrimental differentiation pathway in patients with SLE.

Our results do not exclude negative selection as a complementary mechanism of tolerance. By analogy with mouse studies, several processes including follicular exclusion, lack of $\mathrm{T}$ cell help, desensitization of antigen receptor signaling due to chronic occupancy by self-antigen, and Fas-dependent killing by $\mathrm{CD}^{+} \mathrm{T}$ cells could be involved in the censoring of autoreactive VH4-34 N cells that do not enter the MZ differentiation pathway (11). In fact, negative selection may be required to explain how germinal center and memory VH4-34 cells that escape earlier checkpoints are prevented from differentiating into plasma cells.

In summary, we have defined a B cell population that satisfies the experimental postulates that have allowed the study of B cell tolerance in transgenic animals. These postulates include the presence of intrinsic autoreactivity in a sizable fraction of the B cell repertoire and the availability of specific reagents that permit accurate, quantitative tracking of the distribution and fate of the autoreactive $B$ cells. Our results provide the first example of a human B cell subset that appears to be exquisitely regulated by anatomical and functional compartmentalization and contribute the first delineation of the checkpoints involved in the control of VH4-34 cells. It remains for future studies to explore the specific mechanisms that operate at those checkpoints.

This experimental system should enable investigators to examine in detail the mechanisms of human $\mathrm{B}$ cell tolerance and its malfunction in autoimmune diseases such as SLE.

\section{Acknowledgments}

This work was supported in part by research grants AG14585 (to I. Sanz); AI33195 (to R.A. Insel); and AI40305 and AI46637 (to G.J. Silverman).
1. Burnet, F.M. 1970. The concept of immunological surveillance. Prog. Exp. Tumor Res. 13:1-27.

2. Goodnow, C.C., Adelstein, S., and Basten, A. 1990. The need for central and peripheral tolerance in the B cell repertoire. Science. 248:1373-1379.

3. Adams, E., Basten, A., and Goodnow, C.C. 1990. Intrinsic B cell hyporesponsiveness accounts for self-tolerance in lysozyme/anti-lysozyme double-transgenic mice. Proc. Natl. Acad. Sci. USA. 87:5687-5691.

4. Nemazee, D., et al. 1991. Clonal deletion of autospecific B lymphocytes. Immunol. Rev. 122:117-132.

5. Erikson, J., et al. 1991. Expression of anti-DNA immunoglobulin transgenes in non-autoimmune mice. Nature. 349:331-334.

6. Hartley, S.B., et al. 1991. Elimination from peripheral lymphoid tissues of self-reactive B lymphocytes recognizing membrane-bound antigens. Nature. 353:765-769.

7. Okamoto, M., et al. 1992. A transgenic model of autoimmune hemolytic anemia. J. Exp. Med. 175:71-79.

8. Tsao, B.P., et al. 1992. Failed self-tolerance and autoimmunity in IgG anti-DNA transgenic mice. J. Immunol. 149:350-358.

9. Offen, D., Spatz, L., Escowitz, H., Factor, S., and Diamond, B. 1992. Induction of tolerance to an IgG autoantibody. Proc. Natl. Acad. Sci. USA. 89:8332-8336.

10. Hannum, L.G., Ni, D., Haberman, A.M., Weigert, M.G., and Shlomchik, M.J. 1996. A disease-related rheumatoid factor autoantibody is not tolerized in a normal mouse: implications for the origins of autoantibodies in autoimmune disease. J. Exp. Med. 184:1269-1278.

11. Goodnow, C.C., et al. 1995. Self-tolerance checkpoints in B lymphocyte development. Adv. Immunol. 59:279-368.

12. Radic, M.Z., Erikson, J., Litwin, S., and Weigert, M.B. 1993. Lymphocytes may escape tolerance by revising their antigen receptors. J. Exp. Med. 177:1165-1173.

13. Tiegs, S.L., Russell, D.M., and Nemazee, D. 1993. Receptor editing in self-reactive bone marrow B cells. J. Exp. Med. 177:1009-1020.

14. Satterthwaite, A., and Witte, O. 2000. The role of Bruton's tyrosine kinase in B cell development and function: a genetic perspective. Immunol. Rev. 175:120-127.

15. Zinkernagel, R.M., Nemazee, D., Kouskoff, V., and Lacaud, G. 2000. Assessing the mechanisms that give rise to autoimmunity. Science. 290:11a.

16. Cyster, J.G., Hartley, S.B., and Goodnow, C.C. 1994. Competition for follicular niches excludes self-reactive cells from the recirculating B cell repertoire. Nature. 371:389-395.

17. Sanz, I., et al. 1989. The smaller human VH gene families display remarkably little polymorphism. EMBO J. 8:3741-3748.

18. Pascual, V., et al. 1991. Nucleotide sequence analysis of the $V$ regions of two IgM cold agglutinins. Evidence that the VH4-21 gene segment is responsible for the major cross-reactive idiotype. J. Immunol. 146:4385-4391.

19. Smith, G., Spellerberg, M., Boulton, F., Roelcke, D., and Stevenson, F. 1995. The immunoglobulin VH gene, VH4-21, specifically encodes autoanti-red cell antibodies against the I or i antigens. Vox Sang. 68:231-235.

20. Thompson, K.M., et al. 1991. Human monoclonal antibodies against blood group antigens preferentially express a VH4-21 variable region gene-associated epitope. Scand. J. Immunol. 34:509-518.

21. Pascual, V., et al. 1992. VH restriction among human cold agglutinins. The VH4-21 gene segment is required to encode anti-I and anti-i specificities. J. Immunol. 149:2337-2344.

22. Chapman, C.J., et al. 1993. Autoanti-red cell antibodies synthesized by patients with infectious mononucleosis utilize the VH4-21 gene segment. J. Immunol. 151:1051-1061.

23. Borretzen, M., Chapman, C., Stevenson, F.K., Natvig, J.B., and Thompson, K.M. 1995. Structural analysis of VH4-21 encoded human IgM allo- and autoantibodies against red blood cells. Scand. J. Immunol. 42:90-97.

24. Stevenson, F.K., et al. 1993. Differential usage of an Ig heavy chain variable region gene by human B cell tumors. Blood. 82:224-230.

25. Riboldi, P., et al. 1994. Two acquired immunodeficiency syndrome-associated Burkitt's lymphomas produce specific anti-i IgM cold agglutinins using somatically mutated VH4-21 segments. Blood. 83:2952-2961.

26. Stevenson, F.K., Smith, G.J., North, J., Hamblin, T.J., and Glennie, M.J. 1989. Identification of normal B cell counterparts of neoplastic cells which secrete cold agglutinins of anti-I and anti-i specificity. $\mathrm{Br} . \mathrm{J}$. Haematol. 72:9-15.

27. Isenberg, D., Spellerberg, M., Williams, W., Griffiths, M., and Stevenson, F. 1993. Identification of the 9G4 idiotope in systemic lupus erythematosus. Br. J. Rheumatol. 32:876-882.

28. Stevenson, F.K., et al. 1993. Utilization of the VH4-21 gene segment by anti-DNA antibodies from patients with systemic lupus erythematosus. J. Autoimmun. 6:809-825.

29. van Vollenhoven, R.F., et al. 1999. VH4-34 encoded antibodies in systemic lupus erythematosus: a specific diagnostic marker that correlates 
with clinical disease characteristics. J. Rheumatol. 26:1727-1733.

30. Pascual, V., and Capra, J.D. 1991. Human immunoglobulin heavy-chain variable region genes: organization, polymorphism, and expression. Adv. Immunol. 49:1-74.

31. Pascual, V., and Capra, J.D. 1992. VH4-21, a human VH gene segment overrepresented in the autoimmune repertoire. Arthritis Rheum. 35:11-18.

32. Pascual, V., Widhopf, G., and Capra, J.D. 1992. The human VH repertoire: a restricted set of $\mathrm{VH}$ genes may be the target of immune regulation. Int. Rev. Immunol. 8:147-157.

33. Bhat, N.M., Bieber, M.M., Spellerberg, M.B., Stevenson, F.K., and Teng, N.N. 2000. Recognition of auto- and exoantigens by V4-34 gene encoded antibodies. Scand. J. Immunol. 51:134-140.

34. Potter, K.N., et al. 1993. Molecular characterization of a cross-reactive idiotope on human immunoglobulins utilizing the VH4-21 gene segment. J. Exp. Med. 178:1419-1428.

35. Silverman, G.J., Schrohenloher, R.E., Accavitti, M.A., Koopman, W.J., and Carson, D.A. 1990. Structural characterization of the second major cross-reactive idiotype group of human rheumatoid factors. Association with the VH4 gene family. Arthritis Rheum. 33:1347-1360.

36. Cary, S.P., Lee, J., Wagenknecht, R., and Silverman, G.J. 2000. Characterization of superantigen-induced clonal deletion with a novel clan IIIrestricted avian monoclonal antibody: exploiting evolutionary distance to create antibodies specific for a conserved $\mathrm{V}-\mathrm{H}$ region surface. $J$. Immunol. 164:4730-4741.

37. Harada, Y., et al. 1996. Identification of early plasma cells in peripheral blood and their clinical significance. Br. J. Haematol. 92:184-191.

38. Agematsu, K., et al. 1998. Generation of plasma cells from peripheral blood memory B cells: synergistic effect of interleukin-10 and CD27/CD70 interaction. Blood. 91:173-180.

39. Odendahl, M., et al. 2000. Disturbed peripheral B lymphocyte homeostasis in systemic lupus erythematosus. J. Immunol. 165:5970-5979.

40. Liu, Y.J, de Bouteiller, O., Arpin, C., Durand, I., and Banchereau, J. 1994. Five human mature B cell subsets. Adv. Exp. Med. Biol. 355:289-296.

41. Pascual, V., et al. 1994. Analysis of somatic mutation in five B cell subsets of human tonsil. J. Exp. Med. 180:329-339.

42. Klein, U., Rajewsky, K., and Kuppers, R. 1998. Human immunoglobulin $(\mathrm{Ig}) \mathrm{M}+\mathrm{IgD}+$ peripheral blood B cells expressing the CD27 cell surface antigen carry somatically mutated variable region genes: CD27 as a general marker for somatically mutated (memory) B cells. J. Exp. Med. 188:1679-1689.

43. Kraj, P., Friedman, D.F., Stevenson, F., and Silberstein, L.E. 1995. Evidence for the overexpression of the VH4-34 (VH4.21) Ig gene segment in the normal adult human peripheral blood B cell repertoire. $J$. Immunol. 154:6406-6420.

44. Kraj, P., et al. 1997. The human heavy chain Ig V region gene repertoire is biased at all stages of B cell ontogeny, including early pre-B cells. $J$. Immunol. 158:5824-5832.

45. Stevenson, F.K., Spellerberg, M.B., Jefferis, R., and Mageed, R.A. 1991. Monoclonal gammopathies and autoimmunity. Topics in Aging Research in Europe. 14:33-39.

46. Rettig, M.B., et al. 1996. VH gene usage is multiple myeloma: complete absence of the VH4.21 (VH4-34) gene. Blood. 87:2846-2852.

47. Slifka, M.K., Antia, R., Whitmire, J.K., and Ahmed, R. 1998. Humoral immunity due to long-lived plasma cells. Immunity. 8:363-372.

48. Isenberg, D.A., et al. 1998. Correlation of 9G4 idiotope with disease activity in patients with systemic lupus erythematosus. Ann. Rheum. Dis. 57:566-570.

49. Arpin, C., et al. 1995. Generation of memory B cells and plasma cells in vitro. Science. 268:720-722.

50. Feizi, T., and Monger, E. 1967. Search for I antigen in human tissues. Nature. 216:1025-1026.

51. Childs, R.A., Kapadia, A., and Feizi, T. 1980. Expression of blood group I and $i$ active carbohydrate sequences on cultured human and animal cell lines assessed by radioimmunoassays with monoclonal cold agglutinins. Eur. J. Immunol. 10:379-384.

52. Grillot-Courvalin, C., et al. 1992. An anti-B cell autoantibody from Wiskott-Aldrich syndrome which recognizes i blood group specificity on normal human B cells. Eur. J. Immunol. 22:1781-1788.

53. Bhat, N.M., et al. 1997. Rapid cytotoxicity of human B lymphocytes induced by VH4-34 (VH4.21) gene-encoded monoclonal antibodies, II. Clin. Exp. Immunol. 108:151-159.

54. Stewart, A.K., Huang, C., Long, A.A., Stollar, B.D., and Schwartz, R.S 1992. VH-gene representation in autoantibodies reflects the normal human B cell repertoire. Immunol. Rev. 128:101-122.
55. Gu, H., Tarlinton, D., Muller, W., Rajewsky, K., and Forster, I. 1991 Most peripheral B cells in mice are ligand selected. J. Exp. Med. 173:1357-1371.

56. Arnold, L.W., Pennell, C.A., McCray, S.K., and Clarke, S.H. 1994. Development of B-1 cells: segregation of phosphatidyl choline-specific B cells to the B-1 population occurs after immunoglobulin gene expression. $J$. Exp. Med. 179:1585-1595.

57. Chen, X., Martin, F., Forbush, K.A., Perlmutter, R.M., and Kearney, J.F. 1997. Evidence for selection of a population of multi-reactive B cells into the splenic marginal zone. Int. Immunol. 9:27-41.

58. Levine, M.H., et al. 2000. A B cell receptor-specific selection step governs immature to mature B cell differentiation. Proc. Natl. Acad. Sci. USA. 97:2743-2748.

59. Martin, F., and Kearney, J.F. 2000. Positive selection from newly formed to marginal zone B cells depends on the rate of clonal production, CD19, and btk. Immunity. 12:39-49.

60. Lam, K.P., and Rajewsky, K. 1999. B cell antigen receptor specificity and surface density together determine B-1 versus B-2 cell development. $J$. Exp. Med. 190:471-477.

61. Hayakawa, K., et al. 1999. Positive selection of natural autoreactive B cells. Science. 285:113-116

62. Watanabe, N., et al. 1999. Expression levels of B cell surface immunoglobulin regulate efficiency of allelic exclusion and size of autoreactive B-1 cell compartment. J. Exp. Med. 190:461-469.

63. Hippen, K.L., Tze, L.E., and Behrens, T.W. 2000. CD5 maintains tolerance in anergic B cells. J. Exp. Med. 191:883-890.

64. Mageed, R.A., et al. 1991. Selective expression of a VHIV subfamily of immunoglobulin genes in human CD5+ B lymphocytes from cord blood. J. Exp. Med. 174:109-113.

65. Brezinschek, H.P., et al. 1997. Analysis of the human VH gene repertoire. Differential effects of selection and somatic hypermutation on human peripheral $\mathrm{CD} 5(+) / \operatorname{IgM}+$ and $\mathrm{CD} 5(-) / \operatorname{IgM}+\mathrm{B}$ cells. J. Clin. Invest. 99:2488-2501.

66. Kiyoi, H., Naito, K., Ohno, R., and Naoe, T. 1995. Comparable profiles of the immunoglobulin heavy chain complementarity determining region (CDR)-3 in CD5+ and CD5- human cord blood B lymphocytes. Immunol. 85:236-240.

67. Ebeling, S.B., Schutte, M.E., and Logtenberg, T. 1993. Peripheral human CD5+ and CD5- B cells may express somatically mutated VH5- and VH6-encoded IgM receptors. J. Immunol. 151:6891-6899.

68. Kumararatne, D.S., and MacLennan, I.C. 1981. Cells of the marginal zone of the spleen are lymphocytes derived from recirculating precursors. Eur. J. Immunol. 11:865-869.

69. Jacobson, L.B., Longstreth, G.F., and Edgington, T.S. 1973. Clinical and immunologic features of transient cold agglutinin-hemolytic anemia. Am. J. Med. 54:514-521.

70. Griffin, J.P. 1969. Cold agglutinins in pneumonia. Ann. Int. Med. 71:667-668.

71. Oliver, A.M., Martin, F., and Kearney, J.F. 1999. IgMhighCD21high lymphocytes enriched in the splenic marginal zone generate effector cells more rapidly than the bulk of follicular B cells. J. Immunol. 162:7198-7207.

72. Bhat, N.M., Bieber, M.M., Chapman, C.J., Stevenson, F.K., and Teng, N.N. 1993. Human antilipid A monoclonal antibodies bind to human $\mathrm{B}$ cells and the $\mathrm{i}$ antigen on cord red blood cells. J. Immunol. 151:5011-5021.

73. Spellerberg, M.B., Chapman, C.J., Mockridge, C.I., Isenberg, D.A., and Stevenson, F.K. 1995. Dual recognition of lipid A and DNA by human antibodies encoded by the VH4-21 gene: a possible link between infection and lupus. Hum. Antibodies Hybridomas. 6:52-56.

74. Thomas, M.D., et al. 1999. A human monoclonal antibody encoded by the V4-34 gene segment recognises melanoma-associated ganglioside via CDR3 and FWR1. Hum. Antibodies. 9:95-106.

75. Carroll, M.C., and Prodeus, A.P. 1998. Linkages of innate and adaptive immunity. Curr. Opin. Immunol. 10:36-40.

76. Gommerman, J.L., and Carroll, M.C. 2000. Negative selection of B lymphocytes: a novel role for innate immunity. Immunol. Rev. 173:120-130.

77. Martin, F., and Kearney, J.F. 2001. B1 cells: similarities and differences with other B cell subsets. Curr. Opin. Immunol. 13:195-201.

78. Miura, Y., et al. 1998. RP105 is associated with MD-1 and transmits an activation signal in human B cells. Blood. 92:2815-2822.

79. George, J., Gilburd, B., and Shoenfeld, Y. 1997. The emerging concept of pathogenic natural autoantibodies. Hum. Antibodies. 8:70-75.

80. Goodnow, C.C. 1997. Glimpses into the balance between immunity and self-tolerance. Ciba Found. Symp. 204:190-207. 